\title{
Identificação de microrganismos para controle de infecção em feridas crônicas
}

\author{
Identification of microorganisms for infection control in chronic wounds \\ Identificación de microorganismos para el control de infecciones en heridas crónicas
}

Recebido: 17/06/2021 | Revisado: 29/06/2021 | Aceito: 08/07/2021 | Publicado: 18/07/2021

\author{
Gabriella Keren Silva Lima \\ ORCID: https://orcid.org/0000-0001-6912-5985 \\ Universidade Federal de Alagoas, Brasil \\ E-mail: gabriellaklima@ hotmail.com \\ Fabianny Torres de Oliveira \\ ORCID: https://orcid.org/0000-0001-6193-2002 \\ Universidade Federal de Alagoas, Brasil \\ E-mail: fabianny_torres@hotmail.com \\ Thaís Honório Lins Bernardo \\ ORCID: https://orcid.org/0000-0002-8058-8400 \\ Universidade Federal de Alagoas, Brasil \\ E-mail: thais.bernardo@eenf.ufal.br \\ Igor Michel Ramos dos Santos \\ ORCID: https://orcid.org/0000-0001-6557-3369 \\ Universidade de Pernambuco, Brasil \\ E-mail: igor.santos@esenfar.ufal.br \\ Daniel Florencio Ferro \\ ORCID: https://orcid.org/0000-0002-2544-907X \\ Hospital Universitário Professor Alberto Antunes, Brasil \\ E-mail: danielfferro@hotmail.com \\ Larissa Houly de Almeida Melo \\ ORCID: https://orcid.org/0000-0002-6397-1803 \\ Ambulatório de Feridas e Pé Diabético de Arapiraca, Brasil \\ E-mail: larissahouly@ hotmail.com \\ Hilma Keylla de Amorim \\ ORCID: https://orcid.org/0000-0002-5063-4584 \\ Hospital Universitário Professor Alberto Antunes, Brasil \\ E-mail: hilmaamorim30@gmail.com \\ Paulo Sérgio Gomes da Silva \\ ORCID: https://orcid.org/0000-0002-5720-6638 \\ Hospital Universitário Professor Alberto Antunes, Brasil \\ E-mail: enfermagemheha@gmail.com
}

\begin{abstract}
Resumo
Objetivo: identificar os microrganismos presentes em lesões vasculogênicas crônicas, diabéticas e de pressão para o controle de infecções de feridas. Metodologia: Estudo quantitativo, exploratório e descritivo em um hospital público e de ensino de grande porte. Swabs e biópsias foram realizados em pacientes registrados em uma clínica de feridas. Através do resultado da análise microbiológica, os microrganismos foram separados de acordo com os valores de incidência de acordo com a etiologia da lesão. Resultados: Amostra final de 40 pacientes, 40 swabs e 40 biópsias. Dos 40 swabs, 32 foram positivos e 08 negativos; entre as biópsias, 30 foram positivas e 10 negativas. Foram encontrados 15 tipos diferentes de microrganismos, sendo $02(13,3 \%)$ bactérias Gram positivas e 13 Gram negativas. Das bactérias encontradas, o Gram positivo mais frequente foi o Staphylococcus aureus $(8,06 \%)$ produtor de beta-lactamase e o Gram negativo mais comum foi a Pseudomonas aeruginosa (40,3\%). Conclusão: A partir deste estudo foi possível identificar os principais microrganismos de acordo com a etiologia da lesão para o tratamento de infecções.
\end{abstract}

Palavras-chave: Infecção de feridas; Enfermagem; Microrganismos.

\begin{abstract}
Objective: to identify the microorganisms present in chronic vasculogenic, diabetic and pressure lesions for the control of wound infections. Methodology: Quantitative, exploratory and descriptive study in a large public and teaching hospital. Swabs and biopsies were performed on patients registered at a wound clinic. Through the result of the microbiological analysis, the microorganisms were separated according to the incidence values according to the etiology of the lesion. Results: Final sample of 40 patients, 40 swabs and 40 biopsies. Of the 40 swabs, 32 were positive and 08 negative; among biopsies, 30 were positive and 10 negative. Fifteen different types of microorganisms were found, with $02(13.3 \%)$ Gram positive and 13 Gram negative bacteria. Of the bacteria found, the most frequent Gram positive was Staphylococcus aureus (8.06\%) producing beta-lactamase and the most common negative Gram
\end{abstract}


was Pseudomonas aeruginous (40.3\%). Conclusion: From this study it was possible to identify the main microorganisms according to the etiology of the lesion for the treatment of infections.

Keywords: Wound infection; Nursing; Microorganisms.

\begin{abstract}
Resumen
Objetivo: identificar los microorganismos presentes en lesiones crónicas vasculogénicas, diabéticas y por presión para el control de infecciones de heridas. Metodología: Estudio cuantitativo, exploratorio y descriptivo en un gran hospital público y docente. Se realizaron hisopos y biopsias en pacientes registrados en una clínica de heridas. Mediante el resultado del análisis microbiológico, los microorganismos se separaron según los valores de incidencia según la etiología de la lesión. Resultados: muestra final de 40 pacientes, 40 hisopos y 40 biopsias. De los 40 hisopos, 32 fueron positivos y 08 negativos; entre las biopsias, 30 fueron positivas y 10 negativas. Se encontraron quince tipos diferentes de microorganismos, con $02(13,3 \%)$ bacterias Gram positivas y 13 Gram negativas. De las bacterias encontradas, la Gram positiva más frecuente fue Staphylococcus aureus (8.06\%) productora de betalactamasa y la Gram negativa más común fue la Pseudomonas aeruginosa (40.3\%). Conclusión: A partir de este estudio fue posible identificar los principales microorganismos según la etiología de la lesión para el tratamiento de infecciones.
\end{abstract}

Palabras clave: Infección de heridas; Enfermería; Microorganismos.

\title{
1. Introdução
}

As feridas crônicas são interrupções na continuidade do tecido que não consegue progredir no processo de reparação tecidual em uma média de três meses. A cronicidade das lesões está diretamente relacionada a disfunções intrínsecas de ordem patológica ou fisiológica. A incidência é maior na população adulta e idosa e os tipos de lesões que mais se destacam são as lesões por pressão (LPP), úlcera diabética e úlcera vasculogênica crônica (Vieira \& Araújo, 2018).

Nesse sentido, o tratamento revela dificuldades aos profissionais de saúde, em especial ao profissional enfermeiro que está apto a "avaliar, prescrever e executar curativos em todos os tipos de feridas em pacientes sob seus cuidados, além de coordenar e supervisionar a equipe de enfermagem na prevenção e cuidado de pessoas com feridas" (Conselho Federal de Enfermagem, 2018).

Durante o tratamento um dos maiores problemas está relacionado ao surgimento de infecções, pois elas atrasam a cicatrização da lesão e acarretam em prejuízos sistêmicos para o portador (Gonçalves, Rabeh \& Nogueira, 2014). Uma das causas para surgimento das infecções está ligada diretamente a problemas intrínsecos do paciente, como uma perfusão tissular ineficaz que dificulta a migração celular e o aporte nutricional e isso resulta na redução da resistência dos tecidos à infecção (Nogueira et.al., 2015). No entanto, quando este está comprometido, a proliferação da carga microbiana sobrecarrega a resposta do sistema imunológico podendo levar à colonização crítica e até à infeção” (Pedro \& Saraiva, 2012).

O diagnóstico precoce de infecções é uma forma de prevenir e minimizar consequências resultantes do processo infeccioso, além de otimizar o processo de cicatrização. A epitelização eficiente da lesão proporciona ao seu portador qualidade de vida e impacta diretamente na diminuição de gastos hospitalares (Campos et.al., 2016). O diagnóstico microbiológico faz parte do conhecimento científico necessário para tratamento dos processos infecciosos decorrentes do longo processo de cicatrização.

Diante disso, a questão norteadora deste estudo é: quais os microrganismos presentes em lesões crônicas do tipo vasculogênica, diabética e de pressão para o tratamento de infecções em feridas?

Conforme mencionado anteriormente, para o tratamento de infecções necessita-se de uma avaliação clínica e diagnóstica precisas, por isso a análise microbiológica é necessária para conhecer a microbiota da lesão e associar às manifestações clínicas local e sistêmica. Por isso, esse estudo detectou a necessidade de identificar os microrganismos de acordo com a etiologia da lesão para tratamento de infecções em feridas crônicas do tipo vasculogênica, diabéticas e de pressão. 


\section{Metodologia}

Trata-se de um estudo quantitativo, de caráter exploratório descritivo realizado em um Ambulatório de Feridas de um hospital geral, público e de ensino localizado no nordeste brasileiro. O estudo foi autorizado pela instituição e aprovado pelo Comitê de Ética em Pesquisa da Universidade Federal de Alagoas (UFAL) sob o número CAAE 93044418.6.0000.5013.

A seleção dos sujeitos da pesquisa aconteceu no período de março a junho de 2019 a partir do cadastro dos pacientes em um ambulatório de feridas e, aos que concordaram em participar, foi disponibilizado o Termo de Consentimento Livre e Esclarecido (TCLE), comprovando sua anuência com a efetivação do estudo e assim a coleta do material microbiológico.

Os critérios de inclusão foram pacientes cadastrados no Ambulatório de Feridas e portadores de lesões crônicas do tipo vasculogênica, por pressão ou diabética. Sendo excluídos do estudo pacientes que não estavam disponíveis no dia da coleta microbiológica e pacientes com resultados microbiológicos sugestivos de contaminação.

Dos 147 pacientes cadastrados no Ambulatório de feridas 102 não atendiam aos critérios de tipo de lesão e disponibilidade no dia de coleta, sendo coletados 45 swabs e biópsias, porém 05 amostras resultaram em contaminação, tendo a amostra final de 40 pacientes.

Para realização das coletas microbiológicas, foi realizada limpeza do leito da lesão com soro fisiológico a $0,9 \%$ e debridamento mecânico, nos casos de presença de necrose, para viabilizar a região de coleta. Primeiramente foi realizada a coleta das lesões por Swab de $150 \mathrm{~mm}$ de comprimento e ponta de Rayon em meio Stuart por técnica estéril, seguida pela biópsia com lâmina $\mathrm{n}^{\mathrm{o}} 11$ com a retirada de no mínimo dois fragmentos de áreas distintas do leito da ferida, colocadas em coletor estéril com 10 mililitro de soro fisiológico $0,9 \%$, conforme orientações do laboratório do hospital.

As amostras foram transportadas em recipiente estéril, sem formalina, logo após a coleta; processadas e analisadas pelo Laboratório de Análises Clínicas da referida instituição. A técnica de biópsia foi realizada com o auxílio de um profissional médico.

Os resultados das análises das culturas microbiológicas das feridas foram disponibilizados com o microrganismo incidente na lesão e o antibiograma por frequência. Os resultados foram tabulados no Microsoft Office Professional Plus Excel® 2016. A estatística para swab e biópsia de feridas foi processada pelo Statistical Package for the Social Sciences® (SPSS) versão 22 por meio da análise por estatística inferencial utilizando-se o teste estatístico qui-quadrado, considerando valor significativo $\mathrm{p}<0,05$.

\section{Resultados}

O número de pacientes que fizeram parte da amostra foi de 40, sendo 26 (65\%) do sexo feminino e 14 (35\%) do sexo masculino. Os pacientes da amostra foram 25 (62,5\%) idosos e 15 (37,5\%) adultos jovens (de 18 a 59 anos). Dos 40 pacientes do estudo, $27(67,5 \%)$ são portadores de úlceras do tipo vasculogênica (venosa, arterial ou mista), 11 (27,5\%) possuem úlcera diabética e, apenas, 2 (5\%) são portadores de lesão por pressão.

Os pacientes foram submetidos à cultura microbiana por técnica swab e biópsia. O número de exames coletados em quatro meses consistiu em oitenta amostras, sendo 40 swabs e 40 biópsias em 40 pacientes.

Foram encontrados 15 tipos de microrganismos diferentes, dispostos na Tabela 1, sendo 02 (13,3\%) bactérias de origem Gram positivas e 13 Gram negativas. No que respeita à análise microbiológica das bactérias encontradas, a Gram positiva com maior frequência foi a Staphylococcus aureus $(n=05)$ produtora de beta-lactamase. Entre as Gram negativas, as mais comuns foram Pseudomonas aeruginosas $(n=25)$, Providencia stuartii $(n=06)$ e Proteus mirabilis $(n=04)$. 
Tabela 1 - Microrganismos identificados nos resultados das culturas por swab e biópsia no período de março a junho de 2019.

\begin{tabular}{|l|c|c|}
\hline \multicolumn{1}{|c|}{ Microrganismo } & Swab \% $(\mathbf{n}=\mathbf{3 2})$ & Biópsia \% $(\mathbf{n = 3 0})$ \\
\hline Pseudomonas aeruginosa & $43,75(\mathrm{n}=14)$ & $36,6(\mathrm{n}=11)$ \\
\hline Providencia stuartii & $9,37(\mathrm{n}=3)$ & $10(\mathrm{n}=3)$ \\
\hline $\begin{array}{l}\text { Staphylococcus aureus } \\
\text { produtor de beta- } \\
\text { lactamase) }\end{array}$ & $6,25(\mathrm{n}=2)$ & $10(\mathrm{n}=3)$ \\
\hline Proteus mirabilis & $6,25(\mathrm{n}=2)$ & $6,6(\mathrm{n}=2)$ \\
\hline Hafnia alvei & $6,25(\mathrm{n}=2)$ & $3,3(\mathrm{n}=1)$ \\
\hline Escherichia coli & $3,12(\mathrm{n}=1)$ & $6,6(\mathrm{n}=2)$ \\
\hline Morganella morganii & $3,12(\mathrm{n}=1)$ & $3,3(\mathrm{n}=1)$ \\
\hline Klebsiella pneumoniae & $6,25(\mathrm{n}=2)$ & - \\
\hline Enterobacter clocae & $3,12(\mathrm{n}=1)$ & $3,3(\mathrm{n}=1)$ \\
\hline Enterobacter aerogenes & - & $6,6(\mathrm{n}=2)$ \\
\hline Citrobacter freundii & $3,12(\mathrm{n}=1)$ & $3,3(\mathrm{n}=1)$ \\
\hline Cedecea davisae & $3,12(\mathrm{n}=1)$ & $3,3(\mathrm{n}=1)$ \\
\hline Acinetobacter baumanii & $3,12(\mathrm{n}=1)$ & $3,3(\mathrm{n}=1)$ \\
\hline Enterococcus faecalis & - & $3,3(\mathrm{n}=1)$ \\
\hline Burkholderia cepacia & $3,12(\mathrm{n}=1)$ & - \\
\hline
\end{tabular}

$\mathrm{n}=\mathrm{n}^{\mathrm{o}}$ de microrganismos.

Fonte: Dados da pesquisa (2020).

Com relação aos tipos de microrganismos encontrados de acordo com a etiologia da lesão, o mais prevalente foi a Gram negativa Pseudomonas aeruginosas, como apresentado na Tabela 2. As Gram positivas foram encontradas apenas nas úlceras vasculogênicas e diabéticas. A maior diversidade de microrganismos foi encontrada em lesões do tipo vasculogênica, correspondendo a 08 tipos de microrganismos distintos. 
Tabela 2 - Microrganismos identificados nas culturas analisadas no período de março a junho de 2019 de acordo com a etiologia da lesão.

\begin{tabular}{|c|c|c|c|}
\hline $\begin{array}{c}\text { Microrganismos } \\
\text { identificados }\end{array}$ & $\begin{array}{c}\text { Úlcera vasculogênica } \\
(\%)\end{array}$ & Úlcera diabética $(\%)$ & $\begin{array}{c}\text { Lesão por pressão } \\
(\%)\end{array}$ \\
\hline $\begin{array}{l}\text { Acinetobacter } \\
\text { baumanii }\end{array}$ & - & 10 & - \\
\hline Burkholderia cepacia & 2,70 & - & - \\
\hline Cedecea davisae & 5,40 & - & - \\
\hline $\begin{array}{l}\text { Enterobacter } \\
\text { aerogenes }\end{array}$ & - & 10 & - \\
\hline Enterobacter clocae & 5,40 & - & - \\
\hline Enterococcus faecalis & - & 5 & - \\
\hline Escherichia coli & - & 10 & 50 \\
\hline Hafnia alvei & 8,10 & - & - \\
\hline Klebsiella pneumoniae & - & 10 & - \\
\hline Morganella morganii & - & 5 & - \\
\hline Proteus mirabilis & 8,10 & - & - \\
\hline Providencia stuartii & 8,10 & 10 & - \\
\hline $\begin{array}{l}\text { Pseudomonas } \\
\text { aeruginosas }\end{array}$ & 48,64 & 30 & 50 \\
\hline $\begin{array}{l}\text { Staphylococcus aureus } \\
\text { (produtor de beta- } \\
\text { lactamase) }\end{array}$ & 10,81 & - & - \\
\hline
\end{tabular}

Fonte: Dados da pesquisa (2020).

Nos resultados também foi apresentado o antibiograma com a relação de antimicrobianos testados, o resultado de sensibilidade ou resistência e o valor da concentração inibitória mínima (MIC). Dentre os 62 resultados positivos, foi avaliado o antibiograma, sendo divididos em sensível e resistente. Considerando como resistente os microrganismos que apresentaram resistência a todos os antimicrobianos de administração por via oral. O microrganismo apresentando maior resistência foi a Gram negativa Pseudomonas aeruginosa, e o que apresentou maior sensibilidade a antimicrobianos foi a bactéria Gram positiva Staphylococcus aureus.

Foram comparados os resultados coletados por técnica swab e biópsia (Tabela 3). Dos 40 swabs, 32 foram positivos e 08 foram negativos; já dentre as biópsias, 30 foram positivas e 10 negativas. Sendo importante ressaltar que 05 dos resultados negativos por biópsia foram precedidos por resultados positivos por swab e todos pertenciam a portadores de lesões vasculogênicas.

Tabela 3 - Resultados das culturas nas técnicas swab e biópsia do período de março a junho de 2019.

\begin{tabular}{lccc}
\hline & SWAB & BIÓPSIA & TOTAL \\
& $\mathrm{n}=40(\%)$ & $\mathrm{n}=40(\%)$ & $\mathrm{N}=80(\%)$ \\
\hline POSITIVO & $32(80)$ & $30(75)$ & $62(77,5)$ \\
NEGATIVO & $8(20)$ & $10(25)$ & $18(22,5)$ \\
\hline
\end{tabular}

$\mathrm{n}=\mathrm{n}^{\mathrm{o}}$ microrganismos

Fonte: Dados da pesquisa (2020).

Entre as técnicas de coleta swab e biópsia não houve diferença estatística ( $\mathrm{p}=0,000)$ das lesões com presença de microrganismo. A pesquisa revelou em um dos dados que a proporção de resultados positivos foi de 3 identificações por swab para 1 por biópsia, ou seja, a cada 3 lesões com presença de microrganismo identificadas por swab, identificou-se apenas 1 por biópsia. Através dos resultados, foi observado que o método swab apresentou maior sensibilidade para identificar a presença de microrganismos em comparação ao método de biópsia. 
A diferença de 02 resultados positivos na coleta por swab foram de pacientes portadores de lesões do tipo vasculogênica e diabética. No total dos exames, $77,5 \%$ dos resultados foram positivos e $22,5 \%$ foram negativos.

\section{Discussão}

O surgimento de feridas crônicas está relacionado ao aumento de patologias crônicas, como diabetes mellitus e doenças cardiovasculares. Para os portadores de lesões, a infecção é a principal complicação devido ao longo tempo de cicatrização, a constante colonização de microrganismos e aos fatores intrínsecos a patologia de base (Santos et.al., 2018).

Diferente dos resultados do presente estudo, a maioria dos estudos traz a Gram positiva Staphylococcus aureus como a mais prevalente nos resultados de análise microbiológica, porém ambos estudos convergem que a Gram negativa Pseudomonas aeruginosas está associada a focos infecciosos mais resistentes e duradouros (Silva et.al., 2018).

A formação de biofilmes está mais associada a bactérias Gram negativas, pois elas possuem uma cápsula protetora que serve como uma barreira extra para células fagocitárias. Além disso, entre as membranas interna e externa existe um espaço periplasmático onde ficam acumuladas algumas endotoxinas que quando liberadas exacerbam o processo de inflamação do hospedeiro. Por esse motivo, bactérias Gram negativas são as mais presentes na formação de biofilmes e conseguem adquirir uma maior resistência a antimicrobianos (Abbas, Lichtman \& Pillai, 2015).

Lesões do tipo vasculogênica são geralmente os focos de bactérias Gram negativas, sendo mais prevalente a bactéria Pseudomonas aeruginosas. Isso pode ser explicado devido a necessidade de procedimentos invasivos que esses pacientes apresentam no decorrer do processo de cicatrização para o tratamento dos acometimentos vasculares.

A identificação dos microrganismos e sua susceptibilidade a antibióticos é importante no planejamento da terapêutica que será adotada para combate a infecção (Silva et.al, 2018). Nos casos de estagnação da lesão, a cultura será decisiva para novas condutas terapêuticas.

Patologias associadas à insuficiência venosa crônica dos membros inferiores e alterações vasculares podem causar o aparecimento de úlceras vasculogênicas e quando não tratados podem levar a cronicidade dessas lesões (Vázquez et.al., 2020). Alterações neuropáticas periféricas associadas à Diabetes pode também levar ao aparecimento de ulcerações de membros inferiores; a dificuldade de cicatrização que a própria patologia trás também é um fator predisponente para que elas se tornem crônicas, além disso, são lesões que quando acometidas por infecções recorrentes pode levar a amputação de membros (Tanaka \& Pires, 2019).

Outro tipo de lesão que devido aos fatores internos do paciente podem se tornar crônicas são as lesões por pressão que estão associadas a fatores de alteração de mobilidade e sensorialidade, idade e nível de consciência, por causa desses fatores, as áreas, normalmente de proeminência óssea, sofrem isquemia e necrose tecidual devido pressão extrínseca e aumento da umidade local (Pìnto, 2016).

Uma das formas de tratamento de infecção em feridas crônicas é por meio do uso de antimicrobianos, e para uma antibioticoterapia eficaz faz necessário o uso de técnicas que explanem as características microbiológicas da ferida (Silva et.al., 2018).

Os resultados apresentados nessa pesquisa demonstram que não houve divergência estatística significativa quanto aos métodos de coleta quando comparados os resultados das culturas microbiológicas. Proporcionalmente, o método swab apresenta maior sensibilidade na identificação de microrganismos. Gjodsbol e col. (2006), apontam que utilizar as amostras de swab são suficientes para o diagnóstico de infecções. Laitano e col. (2008), relataram que a cultura por meio de coleta swab é suficiente para excluir a hipótese de infecção quando o resultado é negativo, pois ela consegue ser eficaz quando não há presença de infecção, porém em alguns casos o swab pode registrar um falso-positivo que é quando o resultado é positivo, mas quando realizada a biópsia o resultado é negativo. Por esse motivo, o estudo recomenda que quando o resultado do swab for 
positivo deve ser realizada a biópsia para confirmação, mas condutas a nível tópico já podem ser adotadas para controle de infecção, apenas o uso de antibióticos precisa de uma confirmação do resultado para que não ocorra um uso inapropriado da medicação.

Um estudo realizado por Becerra e col. (2016), realizaram a modificação da coloração de Gram (eosina e hematoxilina) aumentando o contraste entre bactérias e o hospedeiro. Após coleta de um fragmento do tecido por técnica de biópsia é possível aplicar a coloração no próprio tecido do hospedeiro, sendo eficaz na diferenciação também de Gram negativas e positivas. Eles definiram essa forma de identificação de microrganismos como rápida, econômica e eficiente para a prática clínica e laboratorial.

Segundo Pinto (2016), mesmo sendo realizada a biópsia para análise por cultura, não é garantido o diagnóstico preciso de infecção, segundo seu estudo, ele observou que a maioria das infecções resistentes estão associadas à formação de biofilmes, e que para detecção de biofilme a biópsia precisa estar associada a métodos de avaliação molecular, pois enquanto o método de cultura consegue identificar os microrganismos, cerca de 25 a $30 \%$ das vezes, a análise por métodos moleculares consegue uma taxa de identificação de 80 a $100 \%$.

\section{Conclusão}

O estudo possibilitou identificar os principais microrganismos de acordo com a etiologia da lesão. Dentre os 15 microrganismos encontrados, a Gram negativa Pseudomonas aeruginosa foi a mais prevalente nas lesões vasculogênicas, diabéticas e por pressão. A Gram positiva mais encontrada foi a Staphylococcus aureus produtora de beta lactamase. A maior diversidade de microrganismos foi encontrada na lesão vasculogênica.

Os resultados apresentados sugerem que sejam realizados novos estudos a respeito do perfil microbiológico de feridas segundo a etiologia da lesão, pois devido ao aumento do consumo de antibióticos, as bactérias vão se modificando para sobreviver, mudando assim o perfil microbiológico dos focos infecciosos com o decorrer do tempo.

A respeito do método de coleta para diagnóstico por cultura, o estudo mostrou que não houve diferença significativa nos resultados por swab e biópsia, porém, proporcionalmente, a técnica de coleta por swab se mostrou mais sensível para realizar identificação dos microrganismos.

Identificar os microrganismos segundo a etiologia da lesão permite ao profissional entender o perfil microbiológico de cada paciente para que assim, em conjunto com a equipe, possam ser adotadas medidas de controle de infecção. O uso da cultura como método de diagnóstico, seja por coleta swab ou biópsia, permite segurança com relação ao planejamento de intervenções, permitindo agilidade na cicatrização das feridas.

\section{Agradecimentos}

Agradecimentos ao Ambulatório de Feridas e Estomias do Hospital Universitário Professor Alberto Antunes; Agradecimentos ao Laboratório de Análises Clínicas do Hospital Universitário Professor Alberto Antunes.

\section{Referências}

Abbas A. K, Lichtman A. H., \& Pillai, S. (2015). Imunologia celular e molecular. Elsevier.

Becerra, S. C. Roy, D. C Sanchez, S. J. Christy, R. J. \& Burmeister, D. M. (2016). An optimized staining technique for the detection of Gram positive and Gram negative bacteria within tissue. BMC Res Notes. 9:216. In: https://bmcresnotes.biomedcentral.com/articles/10.1186/s13104-016-1902-0

Campos M. G. C. A. Sousa A. T. O. Vasconcelos J. M. B. Lucena S. A. P. \& Gomes S. K. A. (2016). Feridas complexas e estomias: aspectos preventivos e manejo clínico. Ideia.

Conselho Federal de Enfermagem. (2018). Anexo a Resolução no 0567 do Conselho Federal de Enfermagem, de 29 de janeiro de 2018 (BR) [Internet]. Regulamenta a atuação da Equipe de Enfermagem no Cuidado aos pacientes com feridas. 
Gjodsbol K. Christensen J. J. Karlsmark T. Jorgensen B. Klein B. M. \& Krogfelt K. A. (2006). Multiple bacterial species reside in chronic wounds: a longitudinal study. Int Wound J [Internet]. 3(3):225-31. In: https://onlinelibrary.wiley.com/doi/pdf/10.1111/j.1742-481X.2006.00159.x

Gonçalves M. B. B Rabeh S. A. N. Nogueira P. C. (2014). Terapia Tópica para Ferida Crônica: Recomendações para a Prática Baseada em Evidências. Rev da Associação Brasileira de Estomaterapia: estomias, feridas e incontinências [online]. https://www.revistaestima.com.br/estima/article/view/337

Laitano F. F Arnt R. A Cosner A. M. \& Doncatto L. F. (2008). Estudo comparativo entre o exame de cultura da biópsia e do "swab" cutâneo para o diagnóstico de infecção em pacientes queimados do HPS-Porto Alegre. Rev. Bras. Cir. Plást. 23(3):162-166. In: http://www.rbcp.org.br/details/406/ptBR/estudo-comparativo-entre-o-exame-de-cultura-da-biopsia-e-do--swab--cutaneo-para-o-diagnostico-de-infeccao-em-pacientes-queimados-do-hps-portoalegre

Nogueira G. A. Oliveira B. G. R. B. Santana R. F. \& Cavalcanti A. C. D. (2015) Diagnósticos de enfermagem em pacientes com úlcera venosa crônica: estudo observacional. Revista Eletrônica de Enfermagem [online]. 17(2): 333-339. In: https://revistas.ufg.br/fen/article/view/28782

Paviani E. R Stadnik C. B \& Heinek I. (2004). Estudo da epidemiologia e perfil de sensibilidade da Pseudomonas aeruginosa. Revista In Farma [online]. 15(11/12): 66 - 70. In: https://www.cff.org.br/sistemas/geral/revista/pdf/84/i03-perfil.pdf

Pedro I., \& Saraiva S. (2012). Intervenções de enfermagem na gestão de biofilmes em feridas complexas. Jornal of Aging and Innovation [online]. 1(6). In: http://journalofagingandinnovation.org/pt/volume1-edicao6-2012/biofilmes-em-feridas-complexas/

Pinto, G. P. N. M. (2016) Biofilmes e Feridas Crônicas (Dissertação de Mestrado). Universidade Fernando Pessoa, Porto, Portugal.

Santos K. C. B. Ribeiro G. S. C. Feitosa A. H. C. Silva B. R. S. \& Cavalcante T. B. (2018). Qualidade de vida de pacientes hospitalizados com feridas crônicas. Revista Eletrônica de Enfermagem [online]. 20:e3286. In: https://revistas.ufg.br/fen/article/view/54130

Silva, V. Marcoleta, A. Silva, V. Flores, D. Aparicio, T. Aburto, I. Latrach, C. \& Febré, N. (2018). Prevalence and susceptibility pattern of bacteria isolated from infected chronic wounds in adult patients. Revista Chilena de Infectologia. 35(2): 155-162. In: https://europepmc.org/article/med/29912253

Tanaka C. M., \& Pires A. P. M. (2019). Perfil microbiológico dos pés diabéticos infectados. 8(1): 14-18. In: https://www.scielo.br/j/rcbc/a/rPHTb6ZvfYkgd9rcczXdYjf/?lang=pt

Tavares, W. (2001). Manual de antibióticos e quimioterápicos antiinfecciosos. $3^{\mathrm{a}}$ ed. São Paulo: Atheneu.

Vázquez, O. R. S. Díaz, L. C. Mantilla, M. E. T. \& Villalonga, L. E. R. (2020). Tratamientos utilizados en los pacientes hospitalizados por úlceras flebostáticas. Revista Cubana de Angiologia y Cirurgia Vascular. 1: e81. In: http://scielo.sld.cu/scielo.php?script=sci_arttext\&pid=S168200372020000100003

Vieira C. P. B., \& Araújo T. M. E. (2018). Prevalence and factors associated with chronic wounds in older adults in primary care. Rev Esc Enferm USP. 52: e03415. In: https://www.scielo.br/j/reeusp/a/vhRVSFBnrGndry36ZV5GFvz/?lang=en 